\title{
review
}

\section{The complex life of simple sphingolipids}

\author{
Anthony H . Futerman ${ }^{1+} \&$ Yusuf A. H annun² \\ 'Weizmann Institute of Science, Rehovot, Israel, and 'M edical University of South Carolina, Charleston, South Carolina, USA
}

The extensive diversity of membrane lipids is rarely appreciated by cell and molecular biologists. Although most researchers are familiar with the three main classes of lipids in animal cell membranes, few realize the enormous combinatorial structural diversity that exists within each lipid class, a diversity that enables functional specialization of lipids. In this brief review, we focus on one class of membrane lipids, the sphingolipids, which until not long ago were thought by many to be little more than structural components of biological membranes. Recent studies have placed sphingolipidsincluding ceramide, sphingosine and sphingosine-1-phosphate- at the centre of a number of important biological processes, specifically in signal transduction pathways, in which their levels change in a highly regulated temporal and spatial manner. We outline exciting progress in the biochemistry and cell biology of sphingolipids and focus on their functional diversity. This should set the conceptual and experimental framework that will eventually lead to a fully integrated and comprehensive model of the functions of specific sphingolipids in regulating defined aspects of cell physiology.

Keywords: lipids; sphingolipids; second messenger; ceramide; sphingosine-1-phosphate; raft

EM BO reports (2004) 5,777-782. doi:10.1038/sj.embor. 7400208

\section{Why biologists can no longer afford to ignore lipids}

"Cell membranes are crucial to the life of the cell". Thus begins a description of the plasma membrane in one of our favourite textbooks (Alberts et al, 2002). Reading on, we learn that "lipids constitute about $50 \%$ of the mass of most animal cell membranes," and that there are "about $10^{9}$ lipid molecules in the plasma membrane of a small animal cell." But sadly, the space devoted to further discussion of lipids in this book, and it must be said (lest the reader think that we have an axe to grind against this excellent book), in most other cell biology and biochemistry textbooks, is far less than that expected on the basis of their high abundance. Why is this? Is so much known about lipid cell biology that there is little new to discuss? Are lipids too boring to be worthy of significant attention? The answer is probably related to the perceived difficulties of working with membrane lipids and to a lack of appreciation of the

\footnotetext{
${ }^{1}$ Department of Biological Chemistry, Weizmann Instituteof Science, Rehovot 76100, Israel

${ }^{2}$ Department of Biochemistry and M olecular Biology, M edical University of South Carolina,

Charleston, South Carolina 29425-2211, USA

+Corresponding author. Tel: +972 89342704; Fax: +972 89344112 ;

E-mail: tony.futerman@weizmann.ac.il
}

Submitted 21 A pril 2004; accepted 21 June 2004 essential and diverse roles that membrane lipids exert in regulating a wide spectrum of physiological events.

Cell membranes and lipids are indeed crucial to the life of the cell, but as many famous and wise people have said before us, "the devil is in the details". And the details are complicated. Not only are there three main classes of lipids in cell membranes (glycerolipids, sphingolipids and sterols), but also a huge structural variety exists within each class so that hundreds of individual lipid species (and tens of thousands of subspecies) exist within each cell. D oes each individual lipid species have defined functions? How are their levels maintained and regulated? How is their complex intracellular distribution maintained in the light of intracellular vesicle transport between cell membranes? And how are their levels replenished after their turnover in intracellular signalling pathways?

In this short review, we discuss some of this complexity, particularly with respect to one class of membrane lipids-the sphingolipids- which were originally named by J.L.W. Thudichum in 1884 owing to their enigmatic nature. Apparently, Thudichum spent much of his spare time thinking about the sphinx of G reek mythology, and so named the compounds that he isolated after the sphinx because of the riddle of their function. Sphingolipids have come of age in the past couple of decades, mainly owing to two key discoveries that have galvanized the research community. First, sphingolipids are now known to act as both first and second messengers in a variety of signalling pathways, and second, they have vital roles in membrane microdomains, the so-called 'lipid rafts'.

The coming of age of sphingolipids is also reflected in the number of reviews written in this period, particularly during the past five years, and it would be perfectly legitimate for the discerning reader to ask why another is necessary. In contrast to other reviews, we attempt to integrate various aspects of sphingolipid biology that are often viewed as separate entities, namely their biochemistry (that is, their metabolic pathways), their cell biology (where sphingolipids reside in the cell and their modes of intracellular transport) and their roles in signalling (how their turnover is regulated and how this has an impact on cell physiology). After introducing these issues in the next section, we attempt their integration with a view to deriving some basic principles that govern sphingolipid biology and physiology.

\section{Sphingolipids and the diversity of membrane lipids}

As is the case for all membrane lipids, sphingolipids are amphipathic molecules that have both hydrophobic and hydrophilic properties, albeit to varying extents. The hydrophobic region consists of a sphingoid long chain base (normally sphingosine, sphinganine or 
phytosphingosine) to which a fatty acid is attached by an amide bond to carbon 2 (Fig 1). The simplest sphingolipid, ceramide (Merrill, 2002), functions both as a key player in cell signalling and as the precursor of more complex sphingolipids. In contrast to complex sphingolipids, which contain a hydrophilic region (such as phosphate in the case of sphingosine-1-phosphate (S1P) and ceramide-1-phosphate, phosphorylcholine in sphingomyelin, and sugar residues in glycosphingolipids (GSLs)), ceramide has a minimal hydrophilic region comprising two $\mathrm{OH}$ groups. In terms of complexity, at least five different sphingoid bases are known in mammalian cells, more than 20 species of fatty acid (varying in chain length, degree of saturation, and degree of hydroxylation) can be attached to the sphingoid base, and around 500 different carbohydrate structures have been described in GSLs. Although there is often some preference for association of specific components in specific sphingolipids (that is, certain fatty acids are preferentially found in certain GSLs), the number of potential combinations is nevertheless bewildering. Although we are not the first to introduce the 'combinatorial' concept with regard to sphingolipids (Kolter et al, 2002), we stress that such complexity demands highly organized and intricate mechanisms of regulation at both the biochemical and cellular levels, which, for the most part, have not been elucidated.

The best defined area of sphingolipid biology is metabolism, as most of the biochemical pathways of synthesis and degradation have been determined, and most of the enzymes involved have finally succumbed to molecular cloning (Hannun \& Luberto, 2004). Briefly, the first steps in biosynthesis involve formation of the sphingoid base follow ed by attachment of a fatty acid to generate dihydroceramide. At this point, the cis 4-5 double bond is inserted in mammals to generate ceramide, which in turn serves as the precursor for sphingomyelin and other complex sphingolipids that are formed by the addition of specific substituents at the $\mathrm{C}_{1}$-hydroxy position (Fig 1). The breakdown of complex sphingolipids proceeds by stepwise degradation that results in the formation of ceramide, which is itself degraded by the action of a family of ceramidases to regenerate sphingoid bases. These bases serve as substrates for the sphingosine kinases that form S1P.

Sphingolipid metabolism can be regulated at multiple levels, including through the control of enzyme expression, post-translational modifications and allosteric mechanisms. Some of these are celltype specific, either to control which sphingolipids are synthesized during different stages of cell development, or in response to specific signals. By way of example of the emerging relationship between sphingolipid structure, metabolism and function, specific pathways and roles have been suggested for ceramide when it contains different fatty acids. Thus, ceramide containing $\mathrm{C}_{16}$-fatty acid (palmitic acid) might be specifically generated in apoptotic responses (Kroesen et al, 2003). M oreover, it is becoming clear that different genes, albeit part of the same gene family, control which fatty acids are $\mathrm{N}$-acylated to sphingoid bases to generate different ceramide species (Riebeling et al, 2003; Venkataraman et al, 2002), and the tissue distribution of the expression of these genes implies that specific ceramides might have specific functions in different tissues. Hence, although the basic blueprint of the biochemical pathways is well described, much remains to be learned about modes of regulation of individual enzymes, and importantly, how flux through the metabolic pathways, and particularly at branch points where more than one enzyme can act on the same substrate, is regulated. This can be illustrated by again considering ceramide, which is at the centre of the pathways of sphingolipid synthesis and degradation (Hannun \& O beid, 2002). What regulates whether ceramide is shunted to GSLs, or sphingomyelin, or through the action of ceramidases to sphingosine and then to S1P? Are there different intracellular pools of ceramide at different intracellular locations? Are there different pools even within the same organelle, or within the same lipid bilayer? Answers to such sophisticated questions concerning sphingolipid metabolism will provide the conceptual and experimental framework that will determine the future directions and evolution of this field.

Concerning sphingolipid cell biology, much progress has been made over the past decade but large gaps still remain in our knowledge, particularly about the modes and regulation of sphingolipid transport. Ceramide synthesis occurs in the endoplasmic reticulum (ER) and that of sphingomyelin and complex GSLs in the Golgi apparatus (Fig 1). As the hydrophobic nature of ceramide prevents its spontaneous transfer through the cytosol (Venkataraman \& Futerman, 2000), it must travel from the ER to the Golgi apparatus by facilitated mechanisms. This was demonstrated to occur by both vesicular and non-vesicular transport (Fukasawa et al, 1999). Of great importance is the isolation and characterization of a protein, CERT, that transports ceramide between the ER and the Golgi apparatus (Hanada et al, 2003). CERT specifically extracts ceramide from membranes, is necessary for sphingomyelin synthesis, and interestingly, contains a pleckstrin homology $(\mathrm{PH})$ domain to allow its targeting to the Golgi apparatus. The discovery of CERT adds a new level of complexity to our understanding of ceramide biology. At present, nothing is known about whether CERT, or perhaps other putative ceramide-binding proteins, is involved in regulating ceramide levels in any of the intracellular signalling pathways in which it is involved. This latter point is particularly important as evidence is now emerging that sphingolipid metabolism can occur in unexpected subcellular locations. Although this view remains close to heresy for many workers in the field, some evidence suggests that sphingomyelin and other sphingolipids are found in the nucleus and in mitochondria (Fig 1; Bionda et al, 2004), and not just in the sub-fractions of the ER membrane that are tightly associated with these organelles in vitro- the mitochondria-associated ER fraction and the inner leaflet of the ER in the case of nuclei. Ganglioside GD3 (an acidic GSL) has been shown to relocate to mitochondria in response to some inducers of apoptosis ( $M$ alisan \& Testi, 2002). A neutral ceramidase has also been found in mitochondria (El Bawab et al, 2000), and there is evidence for a sphingomyelin cycle in the nucleus (Watanabe et al, 2004). Little, if anything, is known about the dynamic interplay between sphingolipid metabolism in the organelles of the endomembrane system and in other organelles not connected to them by classical vesicular transport pathways. But if ceramide can be delivered from the ER to the Golgi by CERT, then similar pathways could exist to deliver precursors to mitochondria or to the nucleus; however, sphingosine (the precursor of ceramide) shows sufficient aqueous solubility to allow its rapid intermembrane transfer, but this might not be the case for other precursors. Alternatively, a complete subset of biosynthetic and degradative enzymes could exist in these organelles. The answer to this question depends on the use of more sophisticated techniques than those used in the past, which mainly consisted of subcellular fractionation. The availability of antibodies to some of the recently purified enzymes should eventually put this question to rest.

Part of the reason for the recent explosion of interest in sphingolipid biology is the role that these lipids have in intracellular signalling (Fig 2), particularly sphingosine, ceramide, S1P, 

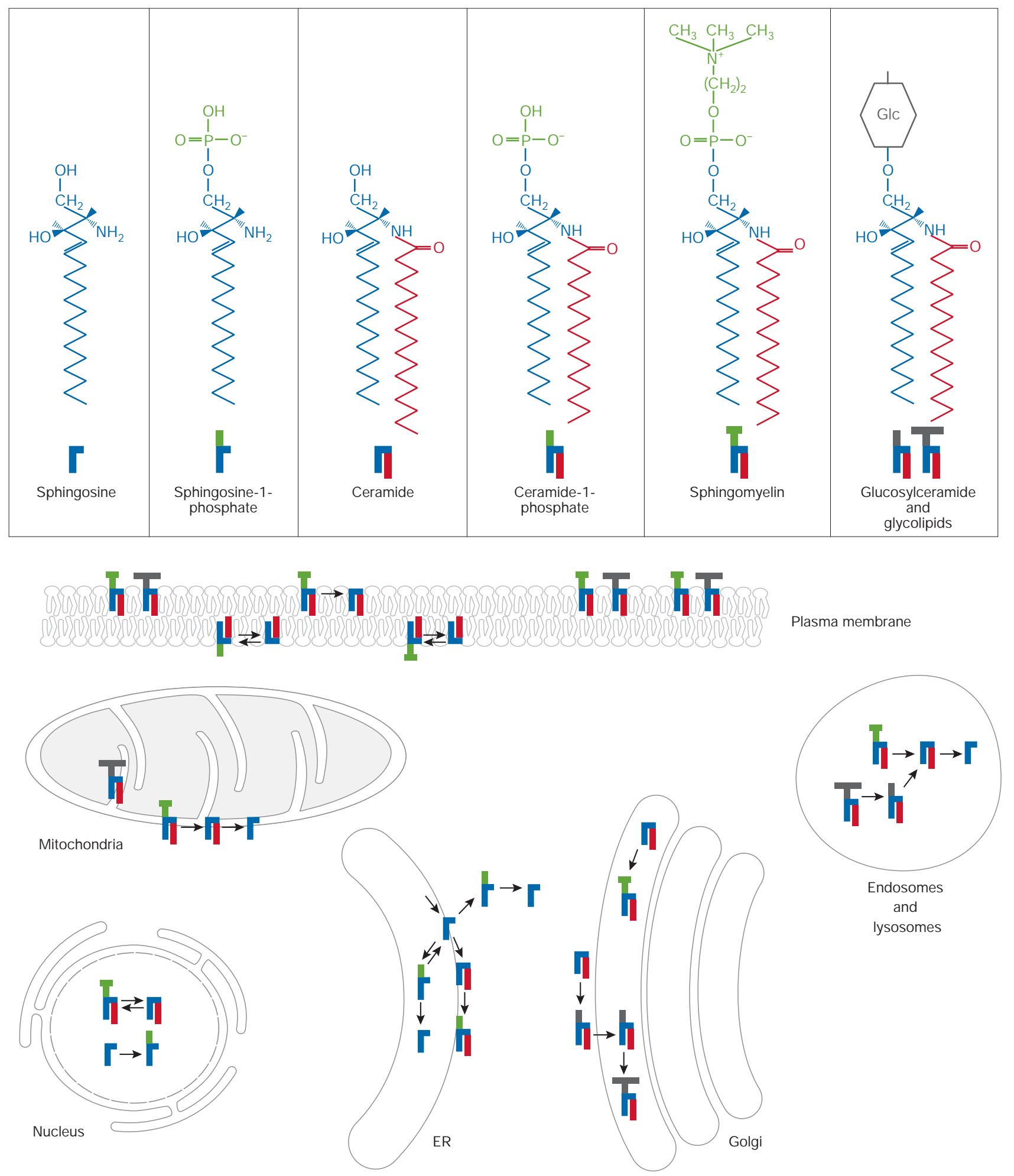

Fig1| The complexity of sphingolipid cell biology. The upper panel shows the structure of some sphingolipids. Notethat, for simplicity, only one kind of sphingoid base (sphingosine, in blue) is shown to which only onekind of fatty acid (palmitic acid, in red) is $\mathrm{N}$-acylated. Similarly, only one glycosphingolipid (GSL) - glucosylceramide (black) - is shown, but sequential addition of other carbohydrate residues results in $>500$ known GSL structures. The bottom panel shows metabolic pathways and the intracellular sites where some of these reactions occur. The topology is uncertain in some cases, particularly for the reactions that occur in the nucleus and mitochondria, and purportedly on the inner leaflet of the plasma membrane. Lipid building blocks are colour coded according to the structures shown above. Transport pathways of the lipids are not shown, but consist of vesicular and non-vesicular pathways. 
ceramide-1-phosphate and sphingosylphosphorylcholine. Indeed the regulatory and signalling functions of individual sphingolipids provide an explanation for the structural diversity of this class of molecules and their intricate pathways of metabolism. Ceramide and SIP are the best-studied 'bioactive' sphingolipids, and they exert opposite effects in many systems, with ceramide usually inhibiting proliferation and promoting apoptosis, and S1P stimulating growth and suppressing apoptosis (Maceyka et al, 2002). Many inducers of apoptosis or grow th arrest activate one or more pathways of ceramide generation, such as sphingomyelinases and the de novo synthesis pathway. In many cases, there is evidence that endogenous ceramide mediates, at least in part, the apoptotic and growth arrest responses of these inducers. For example, both the de novo pathway and neutral sphingomyelinase have been implicated in the actions of tumour necrosis factor- $\alpha$ (TNF- $\alpha$ ). Recently, ceramide has been implicated in the cytotoxic responses to amyloid- $\beta$ peptide, which suggests a role for this pathway in Alzheimer's disease and neurodegeneration (Cutler et al, 2004). Conversely, many growth factors such as platelet-derived growth factor, as well as proinflammatory cytokines such as TNF- $\alpha$, have been shown to activate sphingosine kinase and induce the formation of S1P, which in turn has been implicated in mediating viability and inflammatory responses (Pettus et al, 2003). Therefore, many of the enzymes of sphingolipid metabolism are highly regulated in response to extracellular stimuli, and insight is rapidly accruing on mechanisms that regulate these enzymes. Moreover, great progress has been made in determining the mechanisms of action of sphingosine (such as through the activation of pkh kinases in yeast; Friant et al, 2001), S1P (primarily through the endothelial differentiation gene (EDG)/S1P receptors; Spiegel \& Milstien, 2003) and ceramide (through phosphatases and cathepsin D; Ruvolo, 2003), and such progress should allow the molecular determination of the specific mechanisms and roles of each sphingolipid.

Finally, the importance of sphingolipids in the stability and formation of 'lipid rafts' cannot be ignored. Rafts are segregated microdomains in the lateral plane of the lipid bilayer that are

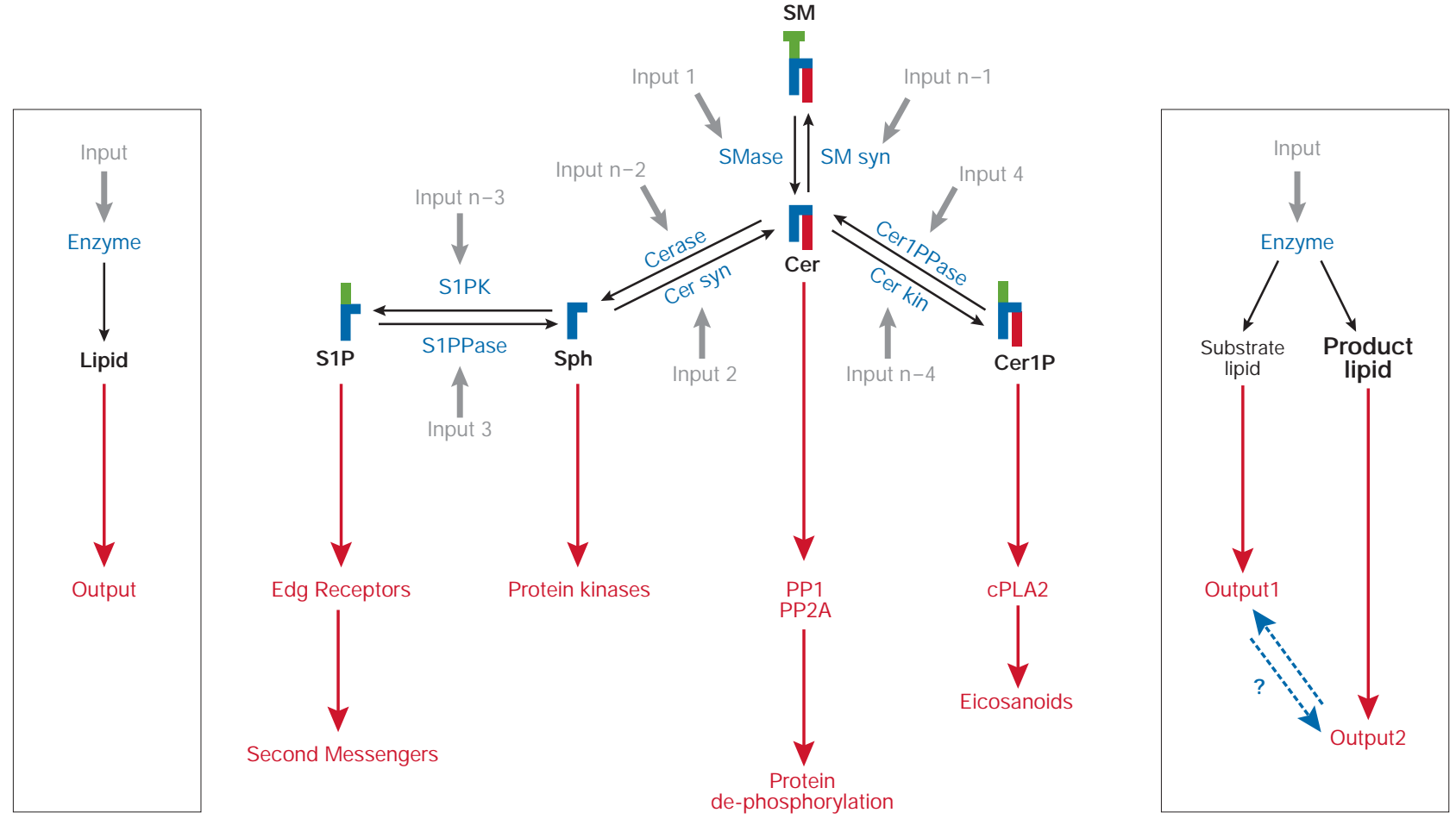

Fig2|The complexity of signalling with simple sphingolipids. In lipid signalling pathways, an input activates (either directly or indirectly) an enzymethat generates a lipid product, which results in an output signal as shown in theinsert on the left of thefigure. In themain panel of thefigure, individual inputs (indicated by positivenumbers when affecting a pathway that leads to ceramide and by negative numbers when affecting a pathway that leads away from ceramide) are shown in grey, theenzymes they affect are in blue, the lipids generated in black, and theoutputs in red. Notethelargediversity of inputs and the relationships between the different lipids generated as illustrated in theinsert on theright, which shows what might happen if both thesubstrateand product aresignalling lipids. This could giverise to diametrically opposed outputs, as indicated by thearrows connecting output 1 and 2 . Notealso that a number of other pathways, and other signalling sphingolipids, arenot shown. M oreover, oneinput may regulatemorethan oneenzyme, and a particular cell may receivemorethan oneinput at a time. Therefore, cell responses would be best described by theintegration of responses to these (and other) pathways, which provides significant flexibility (but also complexity). The challenge in this field is to superimpose Fig 2 on Fig 1 so as to explain how the signalling events in Fig 2 are regulated, modulated and affected by the subcellular localizations at which they occur, and how this is regulated temporally. Lipids are shown using the same simplified structures as in Fig 1. Cer kin, ceramidekinase; Cer syn, ceramide synthase; Cer1PPase, ceramide-1-phosphate phosphatase; CPLA2, phospholipaseA2; ED G receptors, endothelial differentiation genereceptors; SM , sphingomyelin; SM syn, SM synthase; SM ase, sphingomyelinase (of which both neutral and acid act as signalling enzymes); S1PK, sphingosinekinase; S1PPase, sphingosine 1-phosphate phosphatase; PP1, protein phosphatase 1; PP2A, protein phosphatase 2A; cerase, ceramidase. 
enriched in sphingolipids and cholesterol (M unro, 2003). Their formation might be driven, at least in part, by the affinity of cholesterol for specific sphingolipids, although the precise biophysical rules that determine how cholesterol interacts with different sphingolipids have not yet been fully elucidated and may yield surprises (London $\&$ London, 2004). Importantly, with respect to the functional roles of specific sphingolipids, a recent study demonstrated that separate microdomains containing different proteins also contain different subsets of sphingolipids, which differ both in acyl-chain length and the hydrophilic head-group (Brugger et al, 2004). Moreover, it is now increasingly appreciated that plasma membrane rafts might function as platforms for signalling molecules such as receptors and kinases. Significant evidence exists that some of the signalling roles of ceramide are related to its localization to rafts (Gulbins \& Kolesnick, 2003), and the generation of ceramide through the action of sphingomyel inase might actually help in the promotion of raft formation. However, it is also worth pointing out that there is much more to the life of a sphingolipid molecule than its raft association, although this point might not be apparent from reading some of the recent literature.

\section{Integrating metabolism, cell biology and signalling}

Several exciting developments in the sphingolipid universe have recently converged not only in providing new insights into sphingolipid metabolism, biology and regulation, but also in promising to propel the field forward and to allow in-depth deciphering of its complexities. Important among these events has been the molecular identification of all known major enzymes of sphingolipid metabolism, first achieved in yeast in the late 1990s and now in mammals with the recent identification of neutral sphingomyelinase (Karakashian et al, 2004; Marchesini et al, 2003, 2004) and sphingomyelin synthase (H uitema et al, 2004; Yamaoka et al, 2004), two of the last recalcitrant enzymes. Equally as important, the development of advanced mass-spectrometry-based methodologies has allowed the simultaneous assessment of several sphingolipid species as well as the probing of individual subspecies. Deciphering sphingolipid biology has also benefited substantially from other major advances in biological studies, including genomics, proteomics, RNA interference and confocal microscopy. These achievements represent nothing short of a watershed event that should result in deciphering the complexities of sphingolipid biochemistry, molecular biology and physiology. For example, molecular identification of families of enzymes of sphingolipid metabolism has begun to shed light on the subcellular localization of sphingolipids and how this is regulated. Mass spectrometry has clearly revealed that various chain-length species of ceramide have distinct metabolic and functional identities, therefore forcing a more focused approach on individual pathways. For example, to understand 'ceramide biology', it is not only the functions of ceramide that need to be determined, but also the functions of individual species of ceramide and perhaps even the same species in different subcellular compartments and within microdomains in the same membrane.

Conversely, this appreciation of the complexity of sphingolipid metabolism and function, and the emphasis on analysing its molecular constituents and mechanisms, demands the seemingly paradoxical goal of integrating the biology and function of sphingolipids. This integrative approach, best dubbed as '(sphingo)lipidomics', attempts to define the configuration of sphingolipids in any one cell at any one point in time as a stepping stone towards defining the contribution of sphingolipids to the function of a particular cell. This requires understanding the molecular pathways of sphingolipid metabolism, the pathways of sphingolipid signalling, discerning the rules governing the lateral segregation of sphingolipids within the plane of the lipid bilayer (Harder \& van M eer, 2003), their topology across the bilayer and how specific lipids interact with specific protein targets.

Although these are daunting goals, especially when applied to mammalian integrative biology, some of these questions become easier to answer when applied to yeast sphingolipids, whose relative simplicity has allowed the mathematical modelling of sphingolipid pathways in which parameters of sphingolipid metabolism (such as cellular levels, enzyme constants and flux rates) have been integrated into one overall system of equations (Alvarez-Vasquez et al, 2004). Such an approach organizes the rapidly increasing information on sphingolipids (a bioinformatics concept), and also promises to enable predictions of experimental situations (a modelling concept). As these models evolve, they should encompass the effects of compartmentalization of sphingolipid metabolism, microdomain formation, lipid-protein interaction, and any emerging cross-talk betw een pathways of sphingolipid metabolism and signalling and those of other membrane lipids.

\section{ACKN O W LED GEM ENTS}

Work in theFuterman laboratory is supported by the Israel Science Foundation, the European Union, the Children's Gaucher Research Fund and the estate of Louis U ger, Canada. A.H .F. is The Joseph M eyerhoff Professor of Biochemistry at theWeizmann Institute of Science. Work in the Hannun laboratory is supported in part by $\mathrm{N}$ ational Institute of $\mathrm{H}$ ealth grant GM 63265 .

\section{REFEREN CES}

Alberts B, Johnson A, Lewis J, Raff M, Roberts K, Walter P (2002) M olecular Biology of the Cell. Garland Science, N ew York, USA.

Alvarez-Vasquez F, Sims KJ, Hannun YA, Voit EO (2004) Integration of kinetic information on yeast sphingolipid metabolism in dynamical pathway models. J Theor Biol 226: 265-291

Bionda C, Portoukalian J, Schmitt D, Rodriguez-Lafrasse C, Ardail D (2004) Subcellular compartmentalization of ceramide metabolism: MAM and/or mitochondria? Biochem $J$ in press.

Brugger B, Graham C, Leibrecht I, M ombelli E, Jen A, W ieland F, Morris R (2004) The membrane domains occupied by

glycosylphosphatidylinositol-anchored prion protein and Thy-1 differ in lipid composition. J Biol Chem 279: 7530-7536

Cutler RG, Kelly J, Storie K, Pedersen WA, Tammara A, H atanpaa K, Troncoso JC, M attson M P (2004) Involvement of oxidative stress-induced abnormalities in ceramide and cholesterol metabolism in brain aging and Alzheimer's disease. Proc Natl Acad Sci USA 101: 2070-2075

El Bawab S, Roddy P, Q ian T, Bielawska A, Lemasters JJ, Hannun YA (2000) Molecular cloning and characterization of a human mitochondrial ceramidase. Jiol Chem 275: 21508-21513

Friant S, Lombardi R, SchmelzleT, Hall M N, Riezman H (2001) Sphingoid base signalling via Pkh kinases is required for endocytosis in yeast. EM BO J 20: 6783-6792

Fukasawa M, N ishijima M , H anada K (1999) Genetic evidence for ATPdependent endoplasmic reticulum-to-Golgi apparatus trafficking of ceramide for sphingomyelin synthesis in Chinese hamster ovary cells. J Cell Biol 144: 673-685

Gulbins E, Kolesnick R (2003) Raft ceramide in molecular medicine. Oncogene 22: 7070-7077

H anada K, Kumagai K, Yasuda S, Miura Y, Kawano M, Fukasawa M, N ishijima M (2003) M olecular machinery for non-vesicular trafficking of ceramide. Nature 426: 803-809

Hannun YA, Luberto C (2004) Lipid metabolism: ceramide transfer protein adds a new dimension. Curr Biol 14: R163-R165

H annun YA, O beid LM (2002) The ceramide-centric universe of lipidmediated cell regulation: stress encounters of the lipid kind. J Biol Chem 277: $25847-25850$ 
HarderT, van M eer G (2003) Lipid-based membrane domains: physics meets immunology. Traffic 4: 812-820

H uitema K, Van Den Dikkenberg J, Brouwers JF, Holthuis JC (2004) Identification of a family of animal sphingomyelin synthases. EMBO J 23: 33-44

Karakashian AA, Giltiay N V, Smith G M , N ikolova-Karakashian M N (2004) Expression of neutral sphingomyelinase-2 (N SM ase-2) in primary rat hepatocytes modulates IL- $\beta$-induced JN K activation. FASEB J 18: 968-970.

KolterT, Proia RL, Sandhoff K (2002) Combinatorial ganglioside biosynthesis. Biol Chem 277: 25859-25862

Kroesen BJ, Jacobs S, Pettus BJ, Sietsma H, Kok JW, Hannun YA, de Leij LF (2003) BcR-induced apoptosis involves differential regulation of C16 and C24-ceramide formation and sphingolipid-dependent activation of the proteasome. J Biol Chem 278: 14723-14731

London M , London E (2004) Ceramide selectively displaces cholesterol from ordered lipid domains (rafts): implications for lipid raft structure and function. J Biol Chem 279: 9997-10004

M aceyka M, Payne SG, M ilstien S, Spiegel S (2002) Sphingosine kinase, sphingosine-1-phosphate, and apoptosis. Biochim Biophys Acta 1585: 193-201

Malisan F, Testi R (2002) GD 3 ganglioside and apoptosis. Biochim Biophys Acta 1585: 179-187

Marchesini N, Luberto C, Hannun YA (2003) Biochemical properties of mammalian neutral sphingomyelinase 2 and its role in sphingolipid metabolism. J Biol Chem 278: 13775-13783

Marchesini N, O sta W, Bielawski J, Luberto C, O beid LM, Hannun YA (2004) Role for mammalian meutral sphingomyelinase 2 in confluence-induced growth arrest of M CF7 cells. J Biol Chem 279: 25101-25111

Merrill AH Jr (2002) De novo sphingolipid biosynthesis. A necessary, but dangerous, pathway. J Biol Chem 277: 25843-25846

Munro S (2003) Lipid rafts: elusive or illusive? Cell 115: 377-388

Pettus BJ, Bielawski J, Porcelli AM, Reames D L, Johnson KR, M orrow J, Chalfant CE, O beid LM, H annun YA (2003)The sphingosine kinase 1/sphingosine-1-phosphate pathway mediates COX-2 induction and PGE2 production in response to TN F- $\alpha$. FASEB J 17: 1411-1421

Riebeling C, Allegood JC, Wang E, Merrill AH Jr, Futerman AH (2003) Two mammalian longevity assurance gene (LAG 1) family members, trh1 and trh4, regulate dihydroceramide synthesis using different fatty acyl-CoA donors. J Biol Chem 278: 43452-43459

Ruvolo PP (2003) Intracellular signal transduction pathways activated by ceramide and its metabolites. Pharmacol Res 47: 383-392

Spiegel S, M ilstien S (2003) Sphingosine-1-phosphate: an enigmatic signalling lipid. N at Rev Mol Cell Biol 4: 397-407
Venkataraman K, Futerman AH (2000) Ceramide as a second messenger: sticky solutions to sticky problems. Trends Cell Biol 10: 408-412

Venkataraman K, Riebeling C, Bodennec J, Riezman H, Allegood JC, Sullards MC M errill AH Jr, Futerman AH (2002) U pstream of growth and differentiation factor 1 (uog1), a mammalian homolog of the yeast longevity assurance gene 1 (LAG 1), regulates $\mathrm{N}$-stearoyl-sphinganine (C18-(dihydro)ceramide) synthesis in a fumonisin B1-independent manner in mammalian cells. J Biol Chem 277: 35642-35649

Watanabe M, Kitano T, Kondo T, Yabu T, Taguchi Y, Tashima M, U mehara H D omae N, U chiyama T, O kazaki T (2004) Increase of nuclear ceramide through caspase-3-dependent regulation of the "sphingomyelin cycle" in Fas-induced apoptosis. Cancer Res 64: 1000-1007

Yamaoka S, M iyaji M, Kitano T, U mehara H, O kazaki T (2004) Expression cloning of a human cDNA restoring sphingomyelin synthesis and cell growth in sphingomyelin synthase-defective lymphoid cells. J Biol Chem 279: 18688-18693

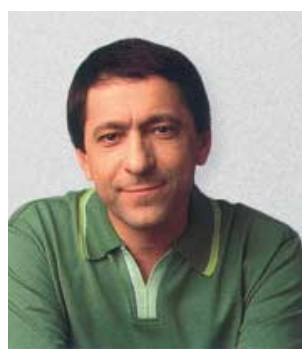

Anthony H . Futerman

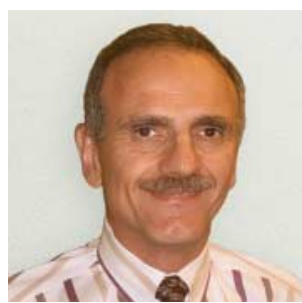

Yusuf A. Hannun 